Zelle, Dennis; Fiedler, Patrique; Haueisen, Jens:

\title{
Artifact reduction in multichannel ECG recordings acquired with textile electrodes
}

Zuerst erschienen in: Biomedical Engineering = Biomedizinische Technik. - Berlin [u.a.] : de Gruyter. - 57 (2012), Suppl. 1, Track-F, p. 171-174.

Erstveröffentlichung: $\quad$ 2012-08-30

ISSN (online): $\quad$ 1862-278X

ISSN (print): 0013-5585

DOI: $\quad 10.1515 / \mathrm{bmt}-2012-4401$

[Zuletzt gesehen: 2019-08-12]

„Im Rahmen der hochschulweiten Open-Access-Strategie für die Zweitveröffentlichung identifiziert durch die Universitätsbibliothek IImenau."

"Within the academic Open Access Strategy identified for deposition by Ilmenau University Library."

„Dieser Beitrag ist mit Zustimmung des Rechteinhabers aufgrund einer (DFGgeförderten) Allianz- bzw. Nationallizenz frei zugänglich."

"This publication is with permission of the rights owner freely accessible due to an Alliance licence and a national licence (funded by the DFG, German

Research Foundation) respectively." 


\title{
Artifact Reduction in Multichannel ECG Recordings Acquired with Textile Electrodes
}

\author{
D. Zelle, P. Fiedler, J. Haueisen \\ Institute of Biomedical Engineering and Informatics, Ilmenau University of Technology, Ilmenau, Germany \\ patrique.fiedler@tu-ilmenau.de
}

\begin{abstract}
Textile electrodes integrated into clothes are an innovative approach for mobile ECG monitoring. However, the lack of electrode fixation on the skin causes high susceptibility to artifacts due to movements and changing electrochemical characteristics of the textile electrodes. In this paper we compare different artifact removal approaches concerning their efficiency in realistic multichannel ECG recordings acquired with textile electrodes. We employed Principal Component Analysis (PCA) and Independent Component Analysis (ICA) in time and frequency domain using FastICA and Temporal Decorrelation Source Separation (TDSEP), respectively. Using textile electrodes comprising silver-coated fibers, five Einthoven-I-leads were acquired during walking, running and extensive breathing. Horizontally aligned electrodes each located on the left and right side of the shoulders, the chest and the back obtain the ECG signals. A reference signal was recorded using self-adhesive $\mathrm{Ag} / \mathrm{AgCl}$ electrodes placed at the inner forearms enabling calculation of the correlation coefficient and the R-peak detection error. The methods using ICA enhance ECG recordings acquired with textile electrodes for all test conditions. TDSEP in the time domain obtains the best results and successfully removes artifacts in recordings of extensive breathing and walking. The results during running show considerable improvements but no complete artifact separation. In conclusion, ICA represents a promising approach for artifact reduction in multichannel ECG recordings acquired with textile electrodes.
\end{abstract}

\section{Introduction}

The evaluation of physical conditions of competitive athletes using physiological parameters is an established standard in sports medicine. Regular sports activity can increase the personal health, particularly strengthening the cardiovascular system [1]. Despite these advantages regular physical activity may be associated with an increased risk of Sudden Cardiac Death (SCD) [2], and therefore the heart and the cardiovascular system are of special interest. Anatomical and physiological disorders imply an abnormal Electrocardiogram (ECG). Hence, ECG is an effective tool in identifying risk factors for SCD.

However, recording the ECG with conventional measurement methods, i.e. using self-adhesive silver/silver chloride $(\mathrm{Ag} / \mathrm{AgCl})$ electrodes is not applicable during exercise because of the distracting movement and application time limitations. A novel approach to measure the electrical heart activity is the use of textile electrodes which include electrically conductive fibers to record the ECG from the body surface. Enabling acquisition of physiological parameters by integration of sensors in clothing has already been successfully achieved for several applications. Borges et al. [3] applied textile electrodes for ECG acquisition in pregnant women. Coosemans et al. [4] integrated woven stainless steel electrodes in a baby suit for continuous ECG monitoring for prevention of sudden infant death syndrome. Paradiso et al. [5] used conductive and piezoresistive yarns to measure simultaneously the ECG, the respiratory signal and the movement activity.
The use of electroconductive fibers has a number of advantages compared to self-adhesive electrodes. The wiring necessary to connect the electrodes to the measurement instrumentation is integrated into clothing and thus the ECG can be transmitted using wireless communication [3-7] or data logging on a small device [8]. Moreover, textile electrodes are reusable, do not need additional electrolyte gel and do not cause skin irritations, thus enabling long-term acquisitions.

However, textile electrodes cannot be attached to the body skin and thus are susceptible to artifacts due to extensive breathing or body movements. Furthermore, electrodes based on electrically conductive fibers are polarizable and show unstable potentials as well as high impedances. The amplitude of resulting artifacts may considerably exceed the ECG signal amplitude thus impeding reliable medical analysis. A possible approach for extracting the ECG signal from highly disturbed recordings is the use of multichannel sensor arrangements in combination with multivariate statistics. Methods like Principal Component Analysis (PCA) [9] and Independent component analysis (ICA) [10-12] are based on multivariate statistics and have been successfully utilized in several signal processing applications on cardiological recordings.

In this paper we compare different techniques based on multivariate statistics concerning their efficiency in removing artifacts from realistic multichannel ECG recordings acquired with textile electrodes. 


\section{Methods}

\subsection{Electrodes and Sensor Arrangement}

Multichannel ECG recordings were acquired with textile electrodes comprising silver-coated synthetic fibers integrated in nonconductive fabrics. The size of each textile electrode is approximately $1.6 \times 15 \mathrm{~cm}$. The material is washable and allows multiple uses. Figure 1 shows an example of the textile electrodes used in this study.

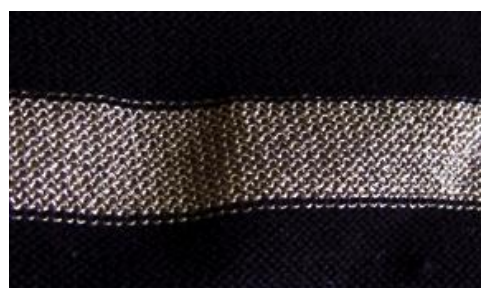

Figure 1 Textile electrodes consisting of electroconductive fibers integrated in knitted fabrics.

In order to obtain five Einthoven-I-leads ten horizontally aligned electrodes were located on the left and right side of the shoulders, the chest and the back. A reference signal was recorded using self-adhesive $\mathrm{Ag} / \mathrm{AgCl}$ electrodes placed at the inner forearms. Figure 2 illustrates this sensor arrangement. The patient ground was placed at the neck of the subject. (a)

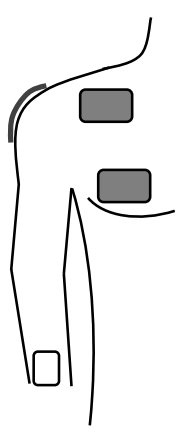

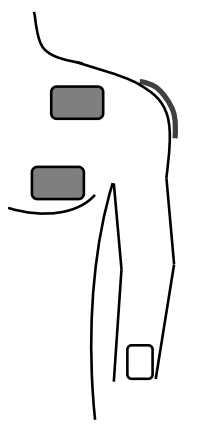

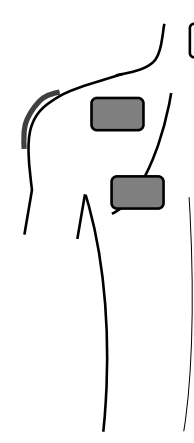

(b)

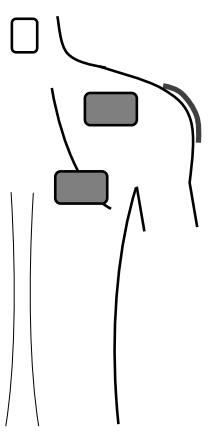

Figure 2 Recording sites for multichannel ECG acquisition using textile electrodes (grey) and selfadhesive $\mathrm{Ag} / \mathrm{AgCl}$ electrodes (white): a) frontal view, and b) rearward view.

\subsection{Implementation}

Applying multivariate statistics to the acquired multichannel ECG enables converting the input data $\boldsymbol{X}$ into a more meaningful representation yielding a matrix $\boldsymbol{Y}$, which contains components that may be associated with the ECG or the artifact signal, respectively. Neglecting the artifact components in $\boldsymbol{Y}$ before reconstructing the ECG signal results in an artifact-free ECG signal. Figure 3 schematically illustrates the use of multivariate statistics in artifact reduction for ECG recordings.
PCA and ICA were implemented in Matlab (Mathworks, Natick, USA) in order to determine $\boldsymbol{Y}$. ICA was solved in both time and frequency domain using the FastICA algorithm [13] and the Temporal Decorrelation Source Separation (TDSEP) algorithm [14]. The number of iterations for the FastICA algorithm was limited to 200. TDSEP was executed with ten correlation matrices timedelayed by $2 \mathrm{~ms}$ each. The transformation from the time to the frequency domain was performed by applying a ShortTime Fourier Transform (STFT) with an FFT-length of 60 samples and a Hanning window of $117 \mathrm{~ms}$ and $90 \%$ overlap.

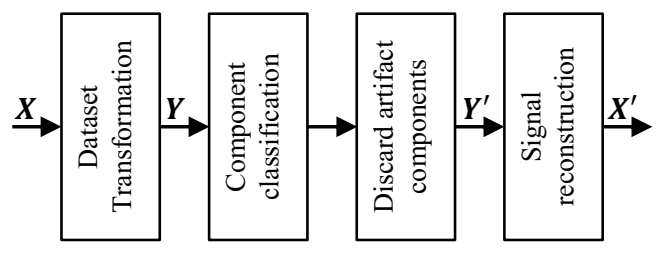

Figure 3 Procedure for artifact reduction in multichannel ECG recordings by applying multivariate statistics.

\subsection{Identification of Artifact Components}

After the input dataset has been transformed into a new representation, the artifact components need to be identified. Exploiting the morphological structure of the ECG the kurtosis as fourth-order moment $\kappa$ of a probability density function (PDF) yields an objective classification parameter. Signals with a Gaussian distribution have $\kappa=3$ (e.g. white noise). Castells et al. [15] showed that PDFs of ECG signals can be assumed to be super-Gaussian with high kurtosis values in the range of $\kappa=16.5 \pm 5.9$, considerably exceeding the values of noise and artifacts. Hence, components with high kurtosis values are assumed to correspond to the ECG signal. Thus, neglecting all other components removes both noise and artifacts and yields to an artifact-free ECG reconstruction.

\subsection{Experimental Setup}

Multichannel ECG recordings were acquired from a healthy male subject during extensive breathing, walking and running using the sensor arrangement shown in Figure 2. The data was recorded using shielded wires connected to a multichannel amplifier (RefaExt, Advanced Neuro Technology B.V., Enschede, Netherlands) with a sampling rate of $512 \mathrm{~Hz}$. The acquired ECG recordings were preprocessed in Matlab using a wavelet decomposition algorithm to remove baseline drift and wavelet denoising to reduce power-line interference and high-frequency noise. The artifact reduction procedure was applied to sections of the ECG recordings with 60 seconds length for each test condition and for interval lengths of two, five and ten seconds. In order to evaluate the performance of the individual techniques the sample correlation coefficient $r$ and the beat detection error $\varepsilon$ were computed with respect to the ECG reference recording. Beat detection was performed using the Pan-Tompkins algorithm [16]. 


\section{Results}

Figure 4 shows the overall results for the correlation coefficient $r$ as boxplots according to the different test conditions. Additionally, the black cross indicates the mean value of the evaluated parameter. The methods using ICA enhance recordings acquired with textile electrodes for all test conditions with respect to the raw ECG signal. The PCA achieves an increase in the correlation to the reference signal for extensive breathing and walking but cannot enhance the raw ECG acquired during running. The results for the beat detection error $\varepsilon$ closely correspond to the results for the correlation coefficient $r$.

(a) Extensive breathing

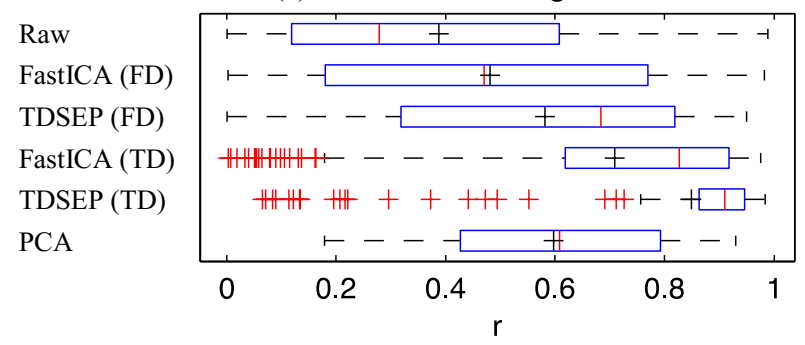

(b) Walking

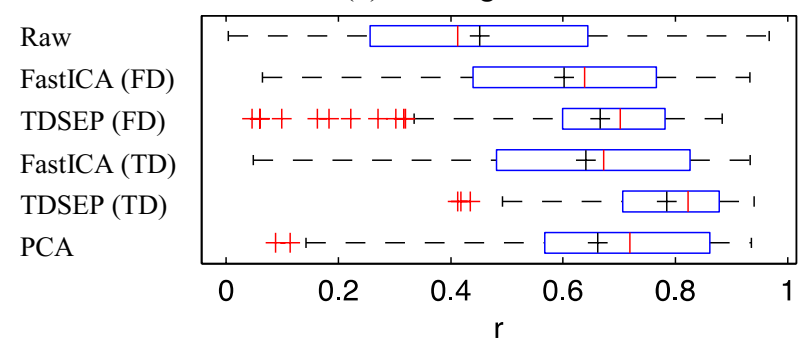

(c) Running

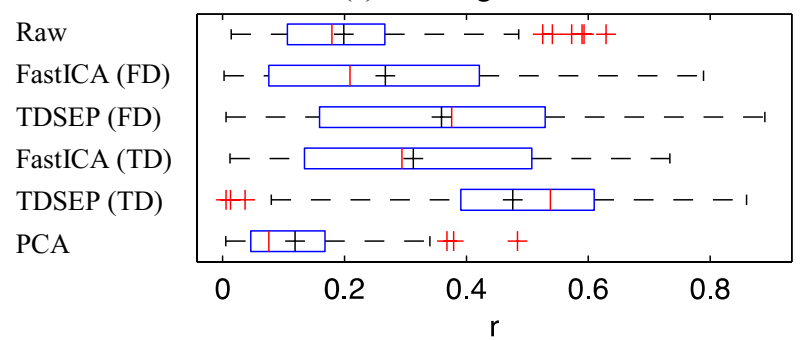

Figure 4 Correlation coefficient $r$ in the time domain (TD) and frequency domain (FD) according to the test conditions extensive breathing, walking, and running.

TDSEP in the time domain produces the best results and successfully removes artifacts in recordings of extensive breathing and walking. After applying TDSEP the mean value for the correlation coefficient increases to 0.85 (raw ECG: 0.39) and $0.79(0.45)$, respectively. The corresponding beat detection errors decrease to $0.10(0.38)$ and $0.06(0.30)$. The results during running show considerable improvements but no complete artifact separation. However, the average correlation increases to $0.48(0.20)$ and the beat detection error decreases to 0.62 (1.17).
Figure 5 shows an example of successful artifact reduction achieved with TDSEP in the time domain for an ECG recording acquired with textile electrodes from the chest. The raw recording after signal conditioning is highly contaminated with motion artifacts considerably exceeding the ECG signal amplitude (Figure 5a). Figure 5b depicts the ICA result using the TDSEP algorithm in the time domain. Comparison with the reference signal acquired with self-adhesive $\mathrm{Ag} / \mathrm{AgCl}$ electrodes in Figure 5c shows the successful artifact reduction as a result of the TDSEP algorithm.

(a) Raw signal

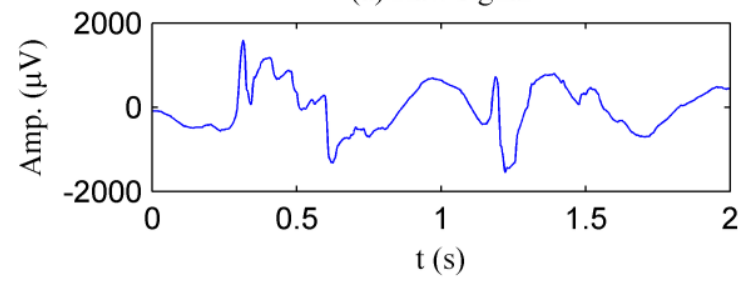

(b) Signal after TDSEP application

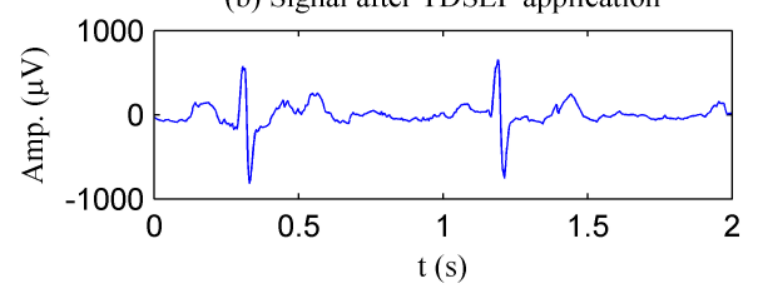

(c) Reference signal

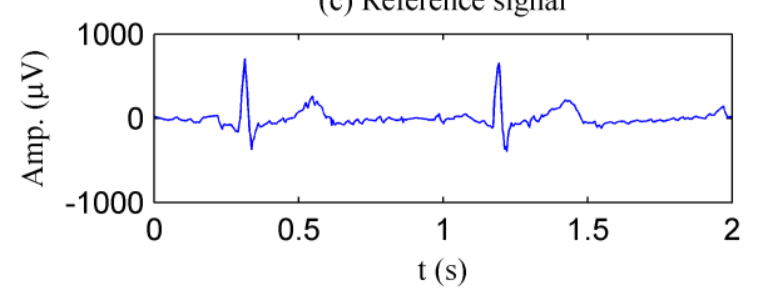

Figure 5 Artifact reduction with TDSEP for an ECG recording from the chest acquired during walking: a) artifact contaminated raw signal, b) signal after TDSEP application, and c) reference signal.

The different test conditions considerably influence the quality of the results. Increasing movement leads to decreasing correlation coefficients and increasing beat detection errors. Furthermore, the results show high dependence on the recording sites implying that the body movements may have a different impact on the signal acquisition at the individual electrode positions. Moreover, the number of channels included in the artifact reduction influences the results for all employed methods. Decreasing the channel number leads to decreasing performance of the artifact removal procedure. In contrast, the interval length does not show any influence on the results indicating that a signal length of two seconds is sufficient for artifact reduction with the investigated methods. 


\section{Conclusion}

Multivariate statistics combined with the temporal structure of the ECG signal, as utilized in TDSEP, allow extracting a reliable ECG from multichannel recordings acquired with textile electrodes even during extensive breathing or walking. For extensive physical activity it will be necessary to further optimize electrode positions and algorithm parameters in order to enable continuous mobile ECG monitoring with electroconductive textiles.

\section{$5 \quad$ References}

[1] Bernadet P: Benefits of Physical Activity in the Prevention of Cardiovascular Diseases. Journal of Cardiovascular Pharmacology, vol. 25, 1995

[2] Corrado D, Migliore F, Bevilacqua M, Basso C, Thiene G: Sudden cardiac death in athletes. Herz, vol. 34, 2009.

[3] Borges LM, Rente A, Velez FJ, Salvado LR, Lebres AS, Oliveira JM, Araujo P, Ferro J: Overview of progress in Smart-Clothing project for health monitoring and sport applications. First International Symposium on Applied Sciences on Biomedical and Communication Technologies, 2008

[4] Coosemans J, Hermans B, Puers R: Integrating wireless ECG monitoring in textiles. The 13th International Conference on Solid-State Sensors, Actuators and Microsystems (TRANSDUCERS '05), 2005

[5] Paradiso R, Loriga G, Taccini N: A Wearable Health Care System Based on Knitted Integrated Sensors. IEEE Transactions on Information Technology in Biomedicine, vol. 9, 2005

[6] Galeottei L, Paoletti M, Marchesi C: Development of a low cost wearable prototype for long-term vital signs monitoring based on embedded integrated wireless module. Computers in Cardiology, 2008

[7] Ottenbacher J, Romer S, Kunze C, Grossmann U, Stork W: Integration of a Bluetooth based ECG system into clothing. Eighth International Symposium on Wearable Computers (ISWC '04), 2004

[8] Pola T, Vanhala J: Textile Electrodes in ECG Measurement. 3rd International Conference on Intelligent Sensors, Sensor Networks and Information (ISSNIP '07), 2007

[9] Castells F, Laguna P, Sörnmo L, Bollmann A, Millet J: Principal Component Analysis in ECG Signal Processing. EURASIP Journal on Advances in Signal Processing, 2007

[10] Castells F, Cebrián A, Millet J: The role of independent component analysis in the signal processing of ECG recordings. Biomedizinische Technik, vol. 52, 2007
Milanesi M, Martini N, Vanello N, Positano V, Santarelli MF, Paradiso R, De Rossi D, Landini L: Multichannel Techniques for Motion Artifacts Removal from Electrocardiographic Signals. 28th Annual International Conference of the IEEE Engineering in Medicine and Biology Society (EMBS '06), 2006

[12] DiPietroPaolo D, Müller HP, Tedeschi W, Park JW, Jung F, Erné SN: Noise reduction in CHD patients by means of BSS. International Congress Series, vol. 1300, 2007

Hyvarinen A: Fast and robust fixed-point algorithms for independent component analysis. IEEE Transactions on Neural Networks, vol. 10, 1999

[14] Ziehe A, Müller KR: TDSEP - an efficient algorithm for blind separation using time structure. Proceedings of the 8th International Conference on Artificial Neural Networks (ICANN'98), 1998

[15] Castells F, Rieta JJ, Millet J, Zarzoso V: Spatiotemporal blind source separation approach to atrial activity estimation in atrial tachyarrhythmias. IEEE Transactions on Biomedical Engineering, vol. 52, 2005

[16] Pan J, Tompkins WJ: A Real-Time QRS Detection Algorithm. IEEE Transactions on Biomedical Engineering, 1985 